\title{
Estudio de la energía y el medio ambiente: una propuesta didáctica computarizada
}

\author{
Edwin Francisco Bonza Camargo \\ Flavio Humberto Fernández Morales \\ Julio Enrique Duarte*
}

Artículo recibido: 26-11-2007 y aprobado: 30-4-2008

Study of the energy and environment: a computerized didactic proposal

Resumen: El objetivo de este proyecto es desarrollar un material educativo computarizado, MEC, para la enseñanza de la energía; contribuyendo de esta manera a solucionar una de las grandes falencias del sistema educativo colombiano: la falta de material didáctico en las aulas de las escuelas y colegios. Además, con la aplicación de este material se aporta a la creación de conciencia en la población escolar hacia un uso más racional de la energía en pro del medio ambiente. El software se diseñó con base en el manejo de lecturas, imágenes, sonidos, videos y animaciones que permiten al estudiante aprender y conceptuar con mayor facilidad sobre un tema tan importante como lo es el de los recursos energéticos renovables y no renovables, abarcando temas tan importantes como la energía solar térmica, que es una posible solución a la crisis energética que se avecina. También, resalta la importancia que tiene la búsqueda de nuevas fuentes de energía que no degraden el medio ambiente.

Palabras clave: Software educativo, educación, energía medio ambiente, didáctica.
Abstract: This project is aimed at developing a Computerized Teaching Software (CTS) for the teaching of energy, contributing in this way to solve one of the biggest problems in the Colombian educational system: the lack of didactic material for schools. The design and implementation of this kind of software, helps teachers to make students aware of the proper use of energy in pro of the environmental care. The software was designed based on an exploratory work that involves readings, images, sounds, video, and animations which allow the students learn and conceptualize easily about renewable and non - renewable energy resources. It also takes into consideration sun energy as a possible solution for a future energy crisis, emphasizing the importance of looking for new energy sources that do not represent damage for the environment.

Key words: Educative software, education, energy, environment, didactics.

Universidad Pedagógica y Tecnológica de Colombia, Facultad Seccional Duitama, Boyacá, Colombia. efraboca@gmail.com; flaviofm1@gmail.com; julioenriqued1@gmail.com 


\section{Introducción}

La educación de hoy se enfrenta a una reorganización, la cual se refleja en el gran deseo de cambio e innovación. Por esta razón la actividad educativa necesita de herramientas que permitan métodos y formas flexibles para la comprensión de los conocimientos e instrumentos para afianzar los conocimientos adquiridos. El sistema educativo involucra principalmente dos agentes: el educando y el educador; estos elementos deben estar relacionados entre sí, buscando como objetivo verificar los procesos y organizar la aprehensión y aplicación de los conocimientos.

La pedagogía moderna implica que el aprendizaje debería ser experimental y útil para la vida cotidiana de los estudiantes. Además, los métodos utilizados deben permitirle actuar de forma autónoma en el proceso de apropiación del conocimiento. Los materiales didácticos deben ser manipulados directamente para lograr un aprendizaje individual de acuerdo con las características únicas de cada persona, a la vez que se refuerza la experimentación como base para el conocimiento científico (Feyman, 1987).

Con el uso de las nuevas tecnologías de información y comunicación, TIC, aplicadas a la educación se pretende rescatar toda una serie de valores perdidos del quehacer del docente (Bartolomé, 1994), en la que el maestro formaba y educaba a partir de los conocimientos. El uso de las nuevas tecnologías en el campo educativo, producto de la era informática, se suma a este nuevo proceso con el objetivo de personalizarla.

Durante mucho tiempo, los métodos de instrucción más utilizados fueron las clases presenciales y el estudio de textos.
Sin embargo, con el desarrollo de las TIC surge la posibilidad de utilizar los programas informáticos en el terreno educativo para la enseñanza como una nueva forma de aprendizaje. El software educativo se define genéricamente como cualquier programa computacional, que sirve de apoyo al proceso de enseñar, aprender y administrar el conocimiento.

En forma restringida, el software educativo es un producto tecnológico que se utiliza en contextos educativos, esté o no específicamente diseñado para tal fin, concebido como uno de los materiales que emplean quien enseña y quien aprende para alcanzar determinados propósitos. Además, es un medio de presentación y desarrollo de contenidos educativos, como lo puede ser un libro o un video (Morales, 2004).

En este trabajo se plantea el desarrollo de un material educativo computarizado, MEC, para la enseñanza de temáticas medioambientales. Específicamente, el MEC presenta información sobre la historia, aplicaciones, tipos, ventajas y recomendaciones para un uso racional de la energía. De esta manera se busca formar personas comprometidas en minimizar problemas como el calentamiento global, el efecto invernadero y la escasez de las fuentes de energía no renovables (Cardozo, Duarte y Fernández, 2005). La temática se escogió de la necesidad detectada por un estudio realizado en algunas instituciones de educación básica acerca de los diferentes métodos y didácticas utilizadas por los docentes de las áreas de ciencias (Angarita y Hillon, 2005). Los resultados arrojados evidenciaron la deficiencia de los estudiantes en conceptos científicos, lo cual se verifica también en las bajas 
calificaciones que periódicamente se registran en las Pruebas Saber publicadas en la página Web del Ministerio de Educación Nacional, MEN: http//:menweb. mineducacion.gov.co.

Con base en lo anterior ha podido establecerse que en la educación básica, hasta el momento, no se concientiza al niño de la importancia que tiene el hacer un uso racional de los recursos naturales, como carbón, petróleo, gas natural y uranio, ya que estos son recursos que mediante procesos físicos y/o químicos proporcionan energía, sin tener en cuenta que están agotándose sus reservas; he aquí la importancia de concientizar al estudiante de que existen otras fuentes de energía, que permanecen constantes en el hábitat y son inagotables. Un ejemplo muy claro de estas fuentes de energía es el sol, fuente de energía diaria que podría aprovecharse mejor. Con la aplicación de este material se busca que el usuario se motive a hacer un uso racional de la energía.

A continuación se describe la metodología utilizada para el desarrollo del software, su estructura y principales características. Igualmente se presentan algunos resultados de la utilización del MEC por parte de los estudiantes.

\section{Metodología y desarrollo}

Para contribuir a la solución de la problemática descrita, se elaboró un MEC multimedial en energía. Se trata de una herramienta informática desarrollada para una población específica de niños y adolescentes de educación básica con edades entre 7 y 14 años, para quienes se prevé un nuevo tipo de experiencia educativa que fortalezca el aprendizaje en ciencia y tecnología.
En este trabajo se tuvo en cuenta la metodología propuesta por Galvis (1993), quien plantea que el desarrollo del conocimiento se expresa de manera vivencial, donde el intérprete pueda interactuar con la aplicación de manera visual (método algorítmico) o mediante el proceso de aprestamiento e interacción (método heurístico).

Durante la generación del software se siguieron las etapas típicas para el desarrollo de materiales educativos: análisis, diseño, prueba, ajuste e implementación (Bartolomé, 1994). Se hizo especial énfasis en la solidez del análisis como punto de partida, en el dominio de teorías sustantivas sobre el aprendizaje y la comunicación humana como fundamento para el diseño de los ambientes educativos computarizados y en la evaluación de cada etapa del proceso para lograr los resultados esperados. Además, se contó con una documentación adecuada y suficiente de la actividad en cada etapa para facilitar el mantenimiento y actualización del material.

Un MEC debe ser ameno, de fácil acceso, lleno de animación y colorido, con la información estructurada de tal manera que motive al estudiante a profundizar en la construcción de su propio conocimiento (Gómez et ál., 2006).

Para cumplir este objetivo el software desarrollado cumple los siguientes requisitos:

Orden de complejidad creciente: el estudiante navega en el MEC adquiriendo información de acuerdo con sus conocimientos previos, intereses, habilidades y perspicacia.

Evaluación: el MEC ofrece un método de evaluación interactivo, gradual, acumulable, que permite al estudiante 
confrontar su progreso en la temática de estudio. Busca reducir el sentido de fracaso para que los usuarios se formen una imagen positiva de sí mismos, a la vez que favorece su desarrollo intelectual.

En el manejo del programa no se usan periféricos especiales, como teclado de conceptos y conmutadores, y si no hay impedimento motriz, es preferible el uso del ratón al del teclado ya que para la navegación se han habilitado las teclas más llamativas.

El programa está abierto para que el profesor pueda introducir sus propios dibujos, textos y sonidos. Los profesores, en muchas ocasiones, se encuentran con la necesidad de adaptar los materiales existentes a su realidad diaria y a las necesidades individuales que presentan los estudiantes.

Desde el punto de vista técnico, para el desarrollo del software se utilizó: Microsoft Windows como plataforma, Microsoft Visual Studio Versión 6.0 como lenguaje de programación y Microsoft Access como administrador de base de datos para el desarrollo del formulario de evaluación.

En el desarrollo del software se optó por utilizar Microsoft Visual Basic 6.0, programa que permite interfaz gráfica de usuario (GUI), la cual contiene varias instrucciones, funciones y palabras clave relacionados con la interfaz gráfica de
Windows. El lenguaje de programación Visual Basic incluye aplicaciones para programar en Excel, Access e Internet; su objetivo es crear un programa especial para los diferentes formatos de la aplicación cliente-servidor (Gary, 1998).

Para el manejo de la base de datos de la evaluación se utilizó el programa Microsoft Access, que permite recopilar cualquier tipo de información para su almacenamiento. Access permite colocar un archivo BD para compartir y presentar información todo a través del manejo de tablas discretas relacionadas con la aplicación de campos comunes. En el desarrollo del software educativo se encargará de la base de datos para el formulario de la evaluación.

En la figura 1 se muestra la organización del tema de energía distribuido en cada uno de los niveles de formación considerados. La aplicación propuesta ofrece la estructura teórica del tema de energía relacionando elementos comunes en cada grado al momento de trabajar y evaluar los temarios propuestos. Estos componentes se refieren a los aportes teóricos, criterios y orientaciones que sustentan la enseñanza de este tema, específicamente referida a la aplicación de los conceptos en la enseñanza y aprendizaje de la energía y sus aplicaciones. 


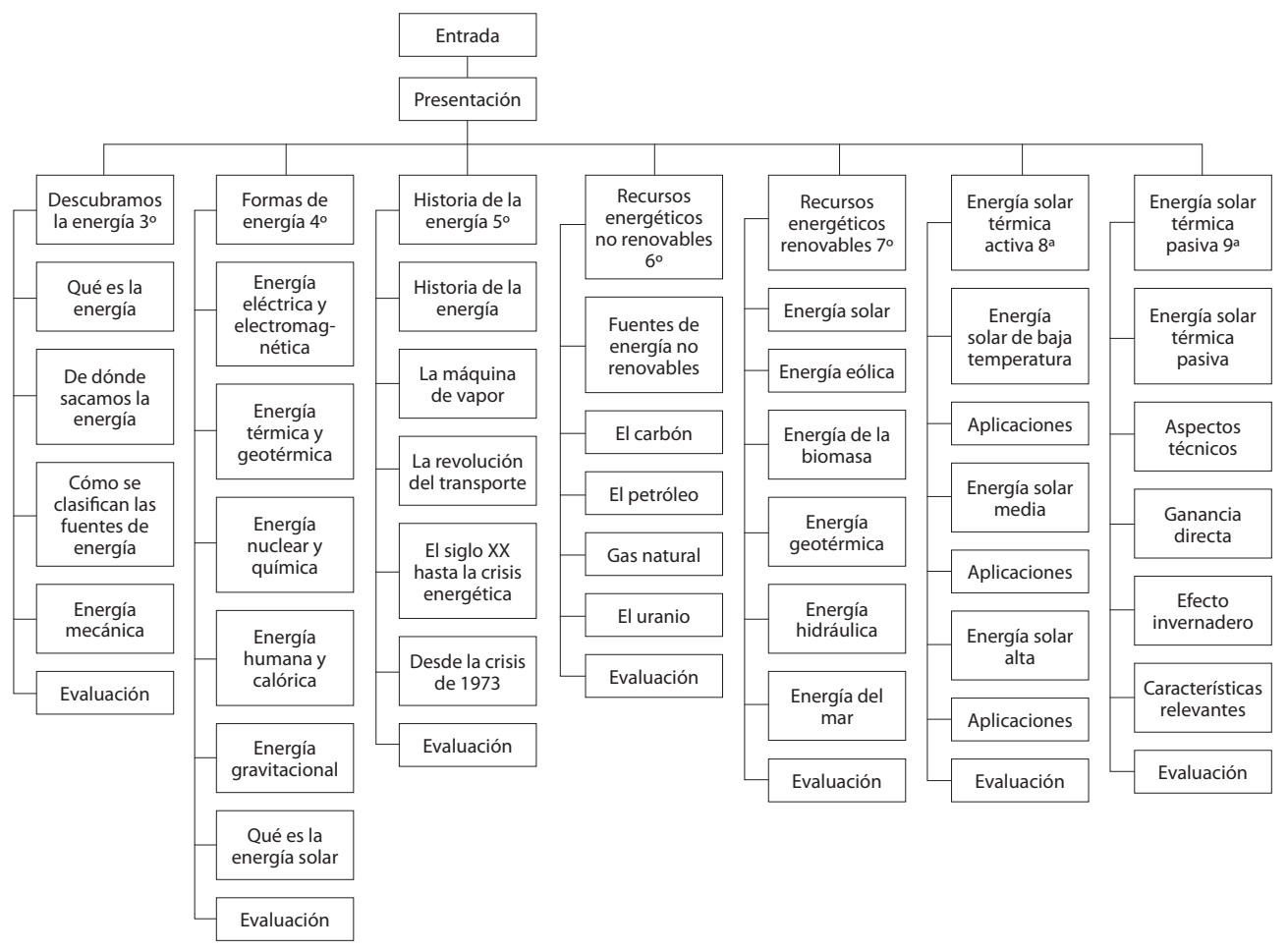

Figura 1. Diagrama lógico de organización del MEC en energía.

La temática a abordar en la enseñanza de la educación básica primaria para el estudio de la energía es: descubramos la energía, formas e historia de la energía, teniendo presente que los grados escogidos para la enseñanza de estas temáticas son tercero, cuarto y quinto. Dentro de los mismos temas encontraremos contenidos como: qué es energía, de dónde sacamos la energía, cómo se clasifican las fuentes de energía, energía mecánica, eléctrica, electromagnética, térmica, geotérmica, nuclear, química, humana, calórica, gravitacional y solar, la máquina de vapor, la evolución del transporte, la crisis energética.

Para el grado sexto se involucran el estudio de los recursos y fuentes de las energías renovables. El estudio de ener- gías renovables como la solar, la eólica y la biomasa, entre otras, son temas a tratar en el grado séptimo de educación básica secundaria. La conceptualización y aplicación de la energía solar térmica activa se ilustra en grado octavo. De igual forma se estudia la energía solar térmica pasiva junto con sus características y aspectos técnicos en el grado noveno, siendo estos los últimos temas tratados en el software. Es de resaltar que la evaluación de las temáticas impartidas en cada grado de formación se estableció al final con el propósito de valorar la información y el impacto formativo que se obtiene con la aplicación del MEC en la enseñanza de la energía y el medio ambiente. 


\section{Resultados}

Este software se creó como ayuda educativa para facilitar la identificación de los conceptos de energías renovables, energías no renovables, energía solar térmica e historia de la energía. El prototipo del MEC fue diseñado para una población objetivo comprendida entre estudiantes de los grados tercero a noveno de educación básica; esto no significa que cualquier otra persona no pueda usarlo. El programa se caracteriza por ser un aplicativo de manejo lineal y por el caudal de información que puede proporcionar. Lo anterior convierte a este programa en una herramienta propicia para apoyar el proceso enseñanzaaprendizaje en los niños y niñas.

Al pasar de la pantalla de inicio se continúa con la presentación, en la cual se hace referencia al tema y grado apropiado para su aplicación. En seguida se llega al menú de contenido principal, donde se visualiza el contenido general referente al software, acompañado de un ícono de ayuda que permite al usuario hacer un mejor manejo del MEC.

Luego de haber identificado el curso se escogerá el tema que quiere estudiarse, en este caso se ha elegido un pantallazo correspondiente al tema: cómo se clasifican las fuentes de energía, preparado para el grado $3 .^{\circ}$, como se muestra en la figura 2 , en la cual se identifica un material multimedia que mezcla el texto con una serie de imágenes alusivas al tema de estudio. En la pantalla se tiene un ícono que permite al estudiante visualizar videos que complementan el tema; también aparece un ícono que permite escuchar la narración de los temas como complemento al material escrito. Los íconos utilizados son llamativos al estudiante y despiertan la curiosidad y la atención por saber qué sucede si se hace clic sobre ellos.

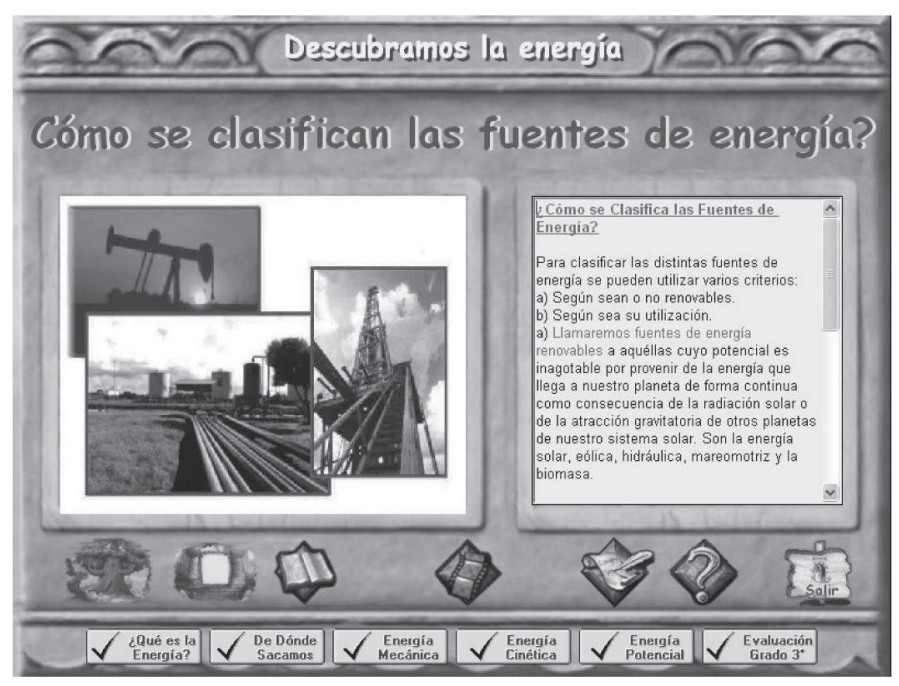

Figura 2. Pantalla típica del MEC de energía. 
En la figura 3 se observa el pantallazo de la evaluación preparada. La evaluación está formada por 10 preguntas, elegidas de forma aleatoria sobre el banco de preguntas disponible. Esta prueba permite establecer el nivel de conocimiento que ha obtenido el estudiante ya que por cada pregunta contestada correctamente se asignan 10 puntos y al contestar la última pregunta el programa arroja el total del puntaje obtenido en múltiplos de 10 hasta 100.

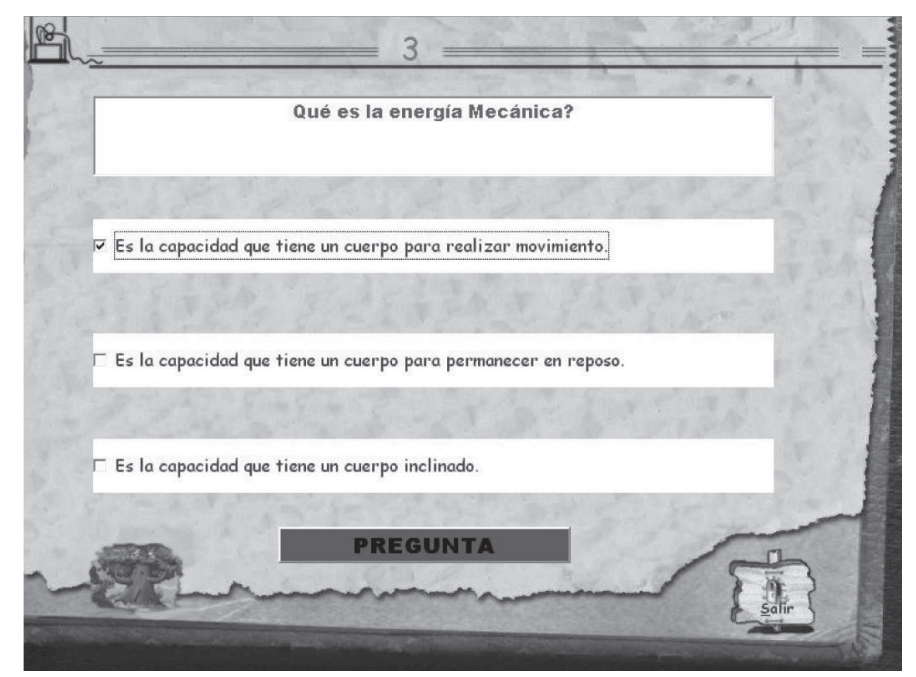

Figura 3. Formato de evaluación.

Se realizó una prueba piloto con el objetivo de evaluar el comportamiento y utilidad del software en un grupo de 35 estudiantes de básica secundaria; al final se indagó a los mismos con respecto a la definición de ayuda didáctica, observándose que el $68,8 \%$ las identifica como material educativo que el docente utiliza para facilitar el aprendizaje, mientras que el restante $32 \%$ las confunde con ciencia y tecnología (véase la figura 4).

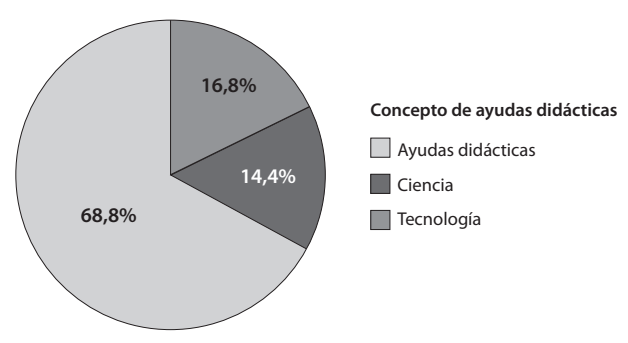

Figura 4. Identificación del concepto de ayuda didáctica. 
Se preguntó a los estudiantes si les agradó manipular el software y recibir la información de la energía y el medio ambiente a través del mismo. En este caso, al 84,4\% de los niños les agradó manipular y utilizar nuevos medios de aprendizaje como el MEC aquí descrito (véase la figura 5).

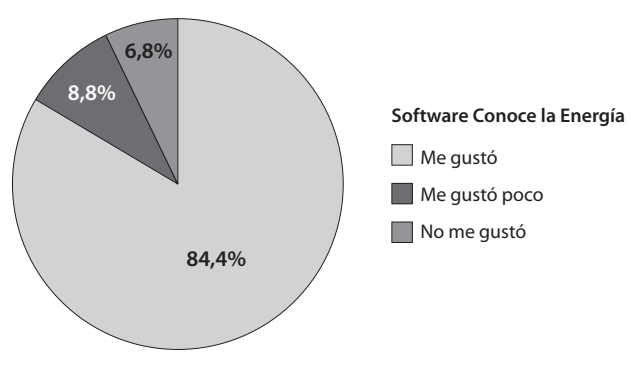

Figura 5. Grado de aceptación en la manipulación y utilización del MEC.

Esta prueba nos permitió validar $y$ verificar el MEC, entendiendo como validación del software el proceso que determina si el programa satisface los requisitos, y verificación como el proceso que determina si las temáticas del grado satisfacen las condiciones del mismo. La estrategia desarrollada en la prueba permitió enfocar con acierto el banco de preguntas para la evaluación y la definición de actividades y conceptos involucrados en cada una de ellas.

Estos resultados descritos son de gran importancia, ya que revelaron el impacto del uso de ayudas didácticas para el aprendizaje de nuevas temáticas relacionadas con el medio ambiente. La prueba permitió verificar que la aplicación de los materiales didácticos, junto con un buen método de enseñanza-aprendizaje, facilita la adquisición del conocimiento por parte de los estudiantes de una manera participativa y lúdica.
Los materiales didácticos son una forma de introducir a los estudiantes en un proceso de enseñanza-aprendizaje novedoso, en el cual los docentes se dan cuenta de la creatividad de los niños, la libertad con que aprenden las cosas, la cooperación para con sus compañeros, a la vez que buscan dar solución a las incógnitas que ellos mismos se generan y que son producto de su entorno.

\section{Conclusiones}

En este trabajo se describió un material educativo computarizado, MEC, para la enseñanza de la energía y conceptos relacionados con el medio ambiente. Con esto se contribuye a solucionar una de las grandes falencias del sistema educativo colombiano: la falta de material didáctico adecuado en las aulas de las escuelas y colegios. Además, con la aplicación de este material se aporta a crear conciencia en la población escolar hacia un uso más racional de la energía en pro del medio ambiente.

El software se diseñó con base en el manejo de lecturas, imágenes, sonidos, videos y animaciones que permiten al estudiante aprender y conceptuar con mayor facilidad sobre un tema tan importante como lo es el de los recursos energéticos renovables y no renovables.

Los resultados obtenidos en la prueba piloto muestran un alto grado de aceptación del MEC por parte de los estudiantes. De esta manera, las ayudas didácticas novedosas se presentan como una alternativa interesante para la enseñanza de temas avanzados que solo se trabajan en cursos superiores, brindándole al estudiante nuevas opciones para su desarrollo académico. 
Además de motivar al estudiante, los materiales educativos computarizados facilitan la labor pedagógica del docente, ya que este tipo de didácticas brindan nuevas posibilidades, permitiéndole cumplir esa labor orientadora exigida en la pedagogía moderna, para que deje de ser un mero transmisor del conocimiento y se convierta en un facilitador del mismo.

Se manejó un concepto integral de formación en el cual la evaluación es parte fundamental del proceso de enseñanza. Para ello se utilizaron formatos de evaluación cuyas preguntas se seleccionan aleatoriamente de una base de datos. Por lo anterior se espera que el MEC tenga efectos positivos en el rendimiento académico y genere un cambio de actitud

\section{Referencias}

Angarita, M. A. y Hillon, N.E. (2005). Cambios que se pueden presentar con la aplicación de ayudas didácticas en la enseñanza de la ciencia y la tecnología en niños. Trabajo de grado, Licenciatura en educación industrial. Duitama: Universidad Pedagógica y Tecnológica de Colombia.

Bartolomé, A. (1994). Multimedia interactivo y sus posibilidades en educación superior. En: http://personal.redestb. es/antoniot/indJ3sc.htm

Cardozo, V. M., Fernández, F. H. y Duarte, J. E. (2005). Diseño y construcción de un calentador solar didáctico. Revista Colombiana de Física, 37(2), 338-348.

Feyman, L. (1987). Lectures on physics. Editorial Addison-Wesley en los estudiantes frente a la aplicabilidad de conocimiento adquirido.

Si bien es cierto que los productos multimediales son de gran importancia en la educación, cabe resaltar que el MEC aquí descrito es una herramienta de apoyo docente y en ningún momento pretende remplazar o reducir la importancia del trabajo de campo.

Los productos multimediales tienen gran importancia en la educación, ya que facilitan la enseñanza, apropiación y comprensión de conceptos complejos, de una manera gráfica, sencilla y amena. Esto confirma el potencial del computador como elemento de apoyo en el proceso enseñanza-aprendizaje, en especial en temas relacionados con el medio ambiente y la energía. $\boldsymbol{\Delta}$

Galvis, A. (1993). Ingeniería del Software Educativo. Bogotá: Ediciones Ugandés.

Gary, C. (1998). Manual de Visual Basic 6.0, Para Windows. Madrid: McGraw Hill.

Gómez, M., Gómez, R., Cardozo, V., Angarita, M., Duarte, J. E. y Fernández Morales, F. H. (2006). Material educativo computarizado para enseñanza de la instrumentación básica en electrónica. VII Congreso de Tecnologías Aplicadas a la Enseñanza de la Electrónica TAEE'06. Madrid, España.

Morales, C., Carmona, V., Espíritu, S. González, I. (2004). Modelo de Evaluación de Software Educativo. Consultado en http://investigacion.ilce.edu.mx/ dice/proyectos/evaluacion/modelo. htm. 